\title{
Dentistry and Intensive Care Unit: A Brief Report
}

\author{
Lisiane Cristina Bannwart ${ }^{1}$ Clóvis Lamartine de Moraes Melo Neto ${ }^{1}$ Daniela Micheline dos Santos ${ }^{1,2}$ \\ André Luiz de Melo Moreno ${ }^{1}$ Aldiéris Alves Pesqueira ${ }^{1}$ Marcelo Coelho Goiato ${ }^{1,2}$ \\ André Pinheiro de Magalhães Bertoz ${ }^{3}$
}

1 Department of Dental Materials and Prosthodontics, School of
Dentistry, São Paulo State University, Aracatuba, São Paulo, Brazil
2 Oral Oncology Center, School of Dentistry, São Paulo State
University, Araçatuba, São Paulo, Brazil
${ }^{3}$ Department of Pediatric and Social Dentistry, School of Dentistry,
São Paulo State University, Araçatuba, São Paulo, Brazil

Eur J Dent 2022;16:449-453.

\begin{abstract}
Address for correspondence Marcelo Coelho Goiato, DDS, MS, PhD, Department of Dental Materials and Prosthodontics, School of Dentistry, São Paulo State University, José Bonifácio, 1193; Araçatuba, São Paulo, ZIP Code: 16050-050, Brazil (e-mail: m.goiato@unesp.br).
\end{abstract}

\section{Abstract \\ Keywords \\ - complete denture \\ - intensive care units \\ - removable partial denture \\ - dental prosthesis \\ - cross infection \\ - acrylic resin}

Objective The aim of this study is to verify whether removable dentures of patients admitted to an intensive care unit (ICU) are niches of microorganisms that can cause pathologies (Staphylococcus aureus, Candida spp., and enterobacteria).

Materials and Methods Fifteen patients who were denture wearers (removable partial denture and complete denture) were included in this study. Patients must wear their dentures daily, and these dentures must have acrylic parts. Microbial biofilm was collected from the acrylic part of one denture of each patient. Then, the biofilm was seeded on different culture media: Sabouraud agar, blood agar, MacConkey agar, and mannitol salt agar. In this study, biochemical evaluations of microorganisms were performed.

Statistical analysis The percentage of dentures with the microorganism identified by each culture medium was calculated.

Results In total, $100 \%$ of the dentures were positive for Staphylococcus spp. (blood agar) and Candida spp. (Sabouraud agar); 33.3\% of the dentures were positive for S. aureus (Mannitol salt agar); and 13.3\% of the dentures were positive for Shigella spp. (MacConkey agar).

Conclusion Removable dentures of patients (removable partial dentures and complete dentures) admitted to an ICU are niches of microorganisms that can cause pathologies.

\section{Introduction}

Intensive care units (ICUs) have contributed to the survival of patients with trauma and other life-threatening conditions. ${ }^{1}$ Despite this, ICUs are associated with a high risk of nosocomial infections. ${ }^{1,2}$

Nosocomial infections are infections that were not present in the patient at the time of admission to a health facility and appear 48 hours after the patient's hospital

published online December 1, 2021
DOI https://doi.org/

10.1055/s-0041-1735797. ISSN 1305-7456. admission. ${ }^{3,4}$ It is estimated that the incidence of these infections is two to five times higher in ICUs than in the general hospitalized population. ${ }^{2}$ According to Ak et al, ICU patients are at increased risk of developing nosocomial infections for a variety of reasons, including presence of underlying diseases, prolonged ICU stay, invasive diagnostic and monitoring procedures performed, impaired host defenses, and colonization by resistant microorganisms. ${ }^{2}$ 
Nosocomial infections can affect any part of the body; however, the respiratory tract is the region most often affected (nosocomial pneumonia). ${ }^{2,5}$ Nosocomial pneumonia can occur when the patient aspirates microorganisms (respiratory pathogens), and these microorganisms reach the lower respiratory tract. ${ }^{6,7}$ Microbial plaque can be a source of respiratory pathogens, and it can be found on teeth and removable dentures. ${ }^{6,7}$

Based on the fact that elderly patients admitted to an ICU may be wearers of removable dentures (removable partial denture and complete denture), ${ }^{8}$ and that aging is associated with an increased risk of hospital-acquired infections ${ }^{9}$; the aim of this study is to verify whether removable dentures of patients admitted to an ICU are niches of microorganisms that can cause pathologies (S. aureus, Candida spp., and enterobacteria).

\section{Materials and Methods}

\section{Ethics Committee}

This study was approved by the ethics committee of the Paulista University (number: 45973115.9.0000.5512). This study included patients admitted to the ICU of the Hospital 28 de Agosto in the city of Manaus (Brazil). This study was conducted between September and December 2017.

Initially, 68 patients admitted to the ICU were selected. After applying the inclusion and exclusion criteria, 15 patients ( 11 men and 4 women) were included in this study.

\section{Inclusion Criteria}

- Men and women over 18 years of age.

- Each patient must wear at least one removable denture (complete denture or removable partial denture).

- Removable dentures must have acrylic parts.

- Patients must wear their removable dentures in the ICU.

- Patients must remain in the ICU for at least 6 days.

- Patients must wear their removable dentures daily.

- No use of antibiotics.

\section{Exclusion Criteria}

- Patients with bronchospasm, altered intracranial pressure, or hemodynamic instability.

- Patients in terminal stage.

- Patients who refused to participate in this study.

\section{Sample Collection}

The team of nurses performed the cleaning of the oral tissue and removable dentures of patients. The patients' oral tissue was cleaned with a sterile gauze moistened with $0.12 \%$ chlorhexidine. Removable dentures were cleaned with sterile water and sterile gauze. There were no dentists working at the hospital.

After cleaning one denture of each patient, the biofilm was collected on the acrylic part of the denture. A sterile cotton swab moistened with sterile saline was rubbed over the acrylic base of the denture for 1 minute. After biofilm collection, the swab was placed in a test tube $(16 \times 160 \mathrm{~mm})$ with $3 \mathrm{~mL}$ of sterile buffered saline solution (phosphate buffered saline), and this set was stored in a thermobox at a temperature between $2^{\circ} \mathrm{C}$ and $8^{\circ} \mathrm{C}$. Then, the collected material was sent to the microbiology laboratory of the Paulista University.

The biofilm was seeded on different culture media: Sabouraud agar, blood agar, MacConkey agar, and Mannitol salt agar. Petri dishes were placed in a laboratory oven at $35^{\circ} \mathrm{C}$, and after 48 hours, the presence or absence of colonies was checked. In this study, biochemical evaluations of microorganisms were performed.

The percentage of dentures with the microorganism identified by each culture medium was calculated.

\section{Results}

The mean age of the participants was approximately 71 years, and the mean time of wearing the same denture or pair of dentures was 10.5 years.

All partially edentulous patients had dental calculus and gingival inflammation. In addition, in $100 \%$ of patients the following characteristics were observed: (1) removable dentures in poor condition (bacterial calculus adhesion on dentures, wear of acrylic teeth, and loss of retention and stability of dentures); (2) patients slept with their dentures removable; (3) tongue coating; and (4) oral tissue with reddened areas, suggesting the presence of candidiasis.

- Table 1 shows that $100 \%$ of the dentures were positive for Staphylococcus spp. (blood agar) and Candida spp. (Sabouraud agar). It was also observed that $33.3 \%$ of the dentures were positive for $S$. aureus (Mannitol salt agar); and $13.3 \%$ of dentures were positive for Shigella spp. (MacConkey agar).

\section{Discussion}

In the present study, teeth and mucosa were cleaned with $0.12 \%$ chlorhexidine. Chlorhexidine can destroy bacteria by breaking their membranes. ${ }^{10}$ Another method that can be used in conjunction with chemical cleaning is mechanical brushing of the teeth, mucosa and tongue. Thus, for ICU patients, it is important that brushing (mechanical cleaning) be performed before chemical cleaning $(0.12 \%$ chlorhexidine) to remove the tongue coating, bacterial plaque and food remains. ${ }^{8}$ In the present study, tongue coating, dental calculus, gingival inflammation and oral tissue with reddened areas were observed in patients, therefore, the presence of a dentist in an ICU could help to prevent or control these situations. According to Miranda et al, biofilm on tooth surfaces, coated tongues, and periodontal disease tend to aggravate patient clinical conditions because they offer an optimal environment for growth of Gram-negative bacteria. ${ }^{8}$ This growth results in more virulent oral microflora. ${ }^{8}$

In this study, it was possible to identify pathogens on removable dentures (-Table $\mathbf{1}$ ). The poor hygiene of a removable denture allows the accumulation of biofilm on it, and this can contribute to the development of several oral and systemic infections. ${ }^{10}$ Thus, cleaning removable 


\begin{tabular}{|c|c|c|c|c|c|c|c|c|c|c|c|c|c|c|c|c|}
\hline 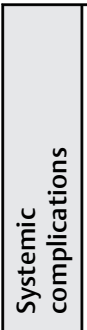 & & 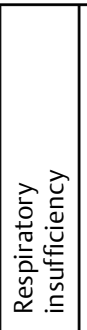 & 离 & 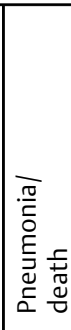 & & 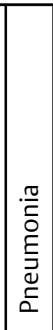 & 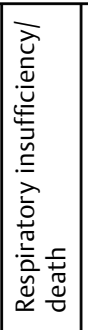 & 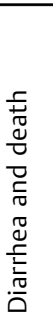 & & 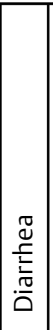 & & & & & & \\
\hline 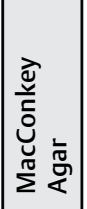 & 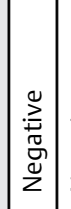 & 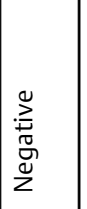 & $\begin{array}{l}\text { 总 } \\
\text { 岕 }\end{array}$ & 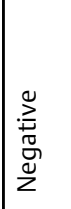 & 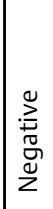 & 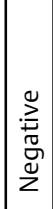 & 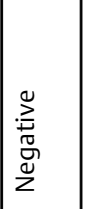 & $\begin{array}{l}\text { 童 } \\
\text { 总 }\end{array}$ & 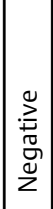 & 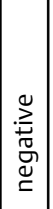 & & & & 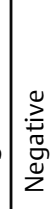 & 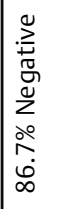 & 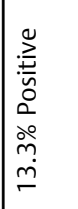 \\
\hline 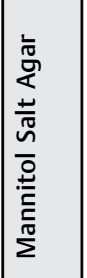 & 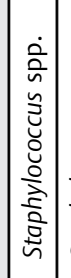 & 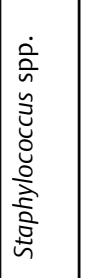 & 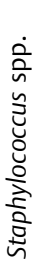 & 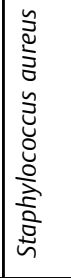 & 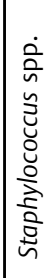 & 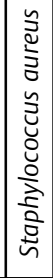 & 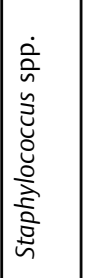 & 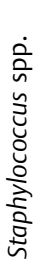 & 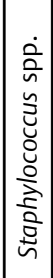 & 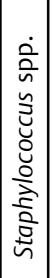 & 气े & 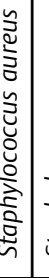 & & ئّ & 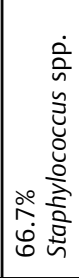 & 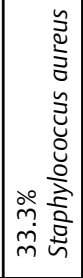 \\
\hline & & & & & & & & & & & & & & & ì & \\
\hline
\end{tabular}

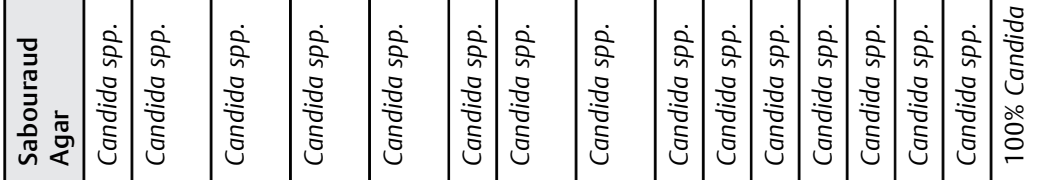

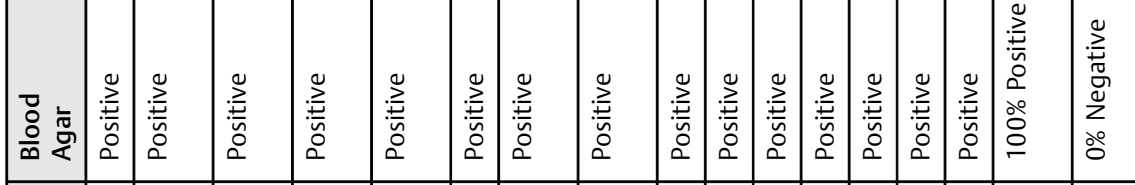

\begin{tabular}{|c|c|c|c|c|c|c|c|c|c|c|c|c|c|c|c|c|}
\hline 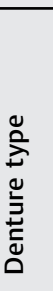 & 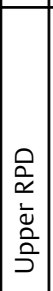 & 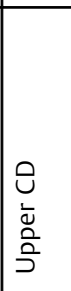 & 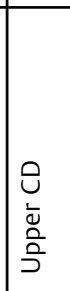 & 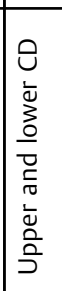 & 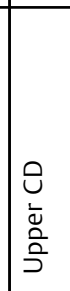 & 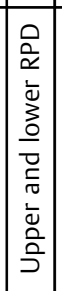 & 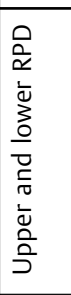 & 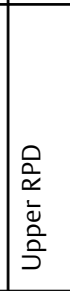 & 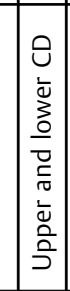 & 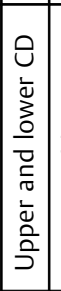 & 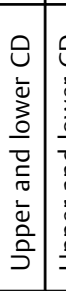 & 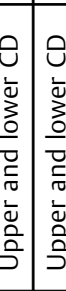 & $\frac{\bar{\sigma}}{\pi}$ & 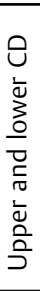 & & \\
\hline & 粒 & 端 & $\frac{\frac{\pi}{0}}{\frac{\pi}{N 5}}$ & 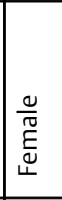 & $\frac{\frac{0}{\pi}}{\Sigma}$ & $\frac{\frac{0}{\pi}}{\frac{2}{2}} \mid$ & $\frac{\frac{\omega}{\tilde{m}}}{\Sigma}$ & \begin{tabular}{|l}
$\frac{\tilde{U}}{\tilde{T}}$ \\
$\underline{\underline{\omega}}$ \\
\end{tabular} & \begin{tabular}{|l}
$\frac{\Delta}{\tilde{\tau}}$ \\
$\frac{\tilde{E}}{\mathrm{E}}$ \\
\end{tabular} & 芯 & $\frac{\frac{\nu}{\pi}}{\Sigma}$ & $\Sigma$ & & & 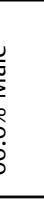 & 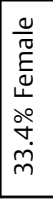 \\
\hline . & - & N & $m$ & $\nabla$ & in & 0 & $\wedge$ & $\infty$ & $\sigma_{0}$ & {$[-1$} & $=$ & $\simeq \cong$ & & & & \\
\hline
\end{tabular}


dentures is important to avoid these problems. ${ }^{10}$ Literature reports that brushing removable dentures with soap, associated with chemical disinfection (e.g., $2 \%$ chlorhexidine) is effective in reducing the colonization of microorganisms (Candida spp. and S. aureus) on them. ${ }^{11-15}$

Patients evaluated in this study slept with their dentures removable. Individuals who sleep with their dentures removable tend to have poor oral hygiene practices, high rates of microbial plaque on their tongues and dentures, oral candidiasis, and visit their dentists less often. ${ }^{16}$ Thus, these individuals may have an increased risk of aspirating microorganisms that can cause pneumonia. ${ }^{16}$ Therefore, this is particularly dangerous for patients admitted to an ICU because their immunity is usually reduced. ${ }^{2}$ This may explain the pneumonia events observed in -Table 1.

Removable dentures (removable partial dentures and complete dentures) should normally be replaced after 5 years of constant use. ${ }^{17,18}$ Over time, the roughness of removable dentures can increase due to their cleaning and daily use, favoring the adhesion of microorganisms. ${ }^{14,19}$ In this study, the mean time of wearing the same denture or pair of dentures was 10.5 years. This suggests that the surface roughness of the evaluated dentures was high.

Patients aged 65 and over are more prone to hospital infections due to generally low immunity, impaired physiological function, chronic illness, and medication use (e.g., immunosuppressants, anticholinergics, sedatives, and gastric acid inhibitors). ${ }^{9}$ Therefore, these patients have a high mortality rate. ${ }^{9}$ In the present study, the mean age of patients was approximately 71 years. ${ }^{9}$ Therefore, advanced age of the evaluated patients was a risk factor.

The most frequent etiologic agents that cause hospital infections are S. aureus, Klebsiella pneumoniae, Pseudomonas aeruginosa, Acinetobacter spp., Escherichia coli, Enterobacter spp., and Candida spp. ${ }^{20-22}$ In this study, two of these microorganisms (S. aureus and Candida spp.) were identified on removable dentures of patients included in this study ( - Table 1 ).

Staphylococci aureus are responsible for skin and soft tissue infections, surgical site infections, endocarditis, pneumonia, and hospital-acquired bacteremia. ${ }^{23,24}$ In this study, $33.3 \%$ of the dentures were positive for S. aureus (Mannitol salt agar). Coincidentally, the wearers of these removable dentures had nosocomial pneumonia (-Table 1). Thus, it is possible that they have aspirated this microorganism causing this systemic complication. ${ }^{8}$

In the ICUs, Candida spp. may be associated with nosocomial infection, ${ }^{25}$ morbidity, and mortality. ${ }^{26}$ All dentures evaluated in this study were positive for Candida spp. It is worth noting that Candida albicans is the most common species found in the mouth. ${ }^{25,27}$ According to Akpan et al, the wearing of dentures produces a microenvironment with low oxygen and low $\mathrm{pH}$, and this favors the growth of Candida spp. $^{27}$ In addition, the ease of adhesion of Candida spp. to acrylic, reduced flow of saliva under the surfaces of the dentures, and lack of oral hygiene can contribute to the development of candidiasis. ${ }^{27}$ Thus, all of this information may explain the presence of Candida spp. in all samples evaluated (-Table $\mathbf{1}$ ).
According to Formal et al, invasive pathogens such as the Shigella spp. damage the intestinal mucosa. ${ }^{28}$ This mucosal damage, which may range from a mild inflammatory response to gross ulceration, is a consequence of this pathogen's ability to invade the intestinal epithelium. ${ }^{28}$ In this study, $13.3 \%$ of dentures were positive for Shigella spp., and their wearers have had diarrhea (systemic complication). Therefore, it is possible that this pathogen caused this systemic complication.

Thus, based on the present study, the dentist is extremely important in an ICU.

\section{Conclusion}

Removable dentures of patients (removable partial dentures and complete dentures) admitted to an ICU are niches of microorganisms that can cause pathologies.

\section{Funding \\ None.}

Conflict of interest

None declared.

\section{References}

1 Maki DG, Crnich CJ, Safdar N. Nosocomial infection in the intensive care unit. Crit Care Med 2008:1003-1069

2 Ak O, Batirel A, Ozer S, Çolakoğlu S. Nosocomial infections and risk factors in the intensive care unit of a teaching and research hospital: a prospective cohort study. Med Sci Monit 2011;17 (05):PH29-PH34

3 Wang L, Zhou KH, Chen W, Yu Y, Feng SF. Epidemiology and risk factors for nosocomial infection in the respiratory intensive care unit of a teaching hospital in China: a prospective surveillance during 2013 and 2015. BMC Infect Dis 2019;19(01):145

4 Divatia JV, Pulinilkunnathil JG, Myatra SN. Nosocomial Infections and ventilator-associated pneumonia in cancer patients. Oncologic Critical Care 2019:1419-1439

5 Vincent JL. Nosocomial infections in adult intensive-care units. Lancet 2003;361(9374):2068-2077

6 Scannapieco FA. Pneumonia in nonambulatory patients. The role of oral bacteria and oral hygiene. J Am Dent Assoc 2006;137 (Suppl):21S-25S

7 Yoneyama T, Yoshida M, Ohrui T, et al; Oral Care Working Group. Oral care reduces pneumonia in older patients in nursing homes. J Am Geriatr Soc 2002;50(03):430-433

8 Miranda AF, de Paula RM, de Castro Piau CG, Costa PP, Bezerra ACB. Oral care practices for patients in intensive care units: a pilot survey. Indian J Crit Care Med 2016;20(05):267-273

9 Avci M, Ozgenc O, Coskuner SA, Olut AI. Hospital acquired infections (HAI) in the elderly: comparison with the younger patients. Arch Gerontol Geriatr 2012;54(01):247-250

10 de Andrade IM, Cruz PC, Silva-Lovato CH, de Souza RF, SouzaGugelmin MC, Paranhos HdeF. Effect of chlorhexidine on denture biofilm accumulation. J Prosthodont 2012;21(01):2-6

11 Salles AE, Macedo LD, Fernandes RA, Silva-Lovato CH, Paranhos HdeF. Comparative analysis of biofilm levels in complete upper and lower dentures after brushing associated with specific denture paste and neutral soap. Gerodontology 2007;24(04): 217-223

12 Moreno A, Dos Santos DM, Lamartine de Moraes Melo Neto C, Luiz de Melo Moreno A, de Magalhães Bertoz AP, Goiato MC. In vitro 
evaluation of the effect of different disinfectants on the biofilm of Staphylococcus epidermidis and Staphylococcus aureus formed on acrylic ocular prostheses. PLoS One 2020;15(10):e0240116

13 Bell JA, Brockmann SL, Feil P, Sackuvich DA. The effectiveness of two disinfectants on denture base acrylic resin with an organic load. J Prosthet Dent 1989;61(05):580-583

14 Felipucci DN, Davi LR, Paranhos HF, Bezzon OL, Silva RF, Pagnano VO. Effect of different cleansers on the surface of removable partial denture. Braz Dent J 2011;22(05):392-397

15 Mima EG, Pavarina AC, Vargas FdaS, Giampaolo ET, Machado AL, Vergani CE. Effectiveness of chlorhexidine on the disinfection of complete dentures colonised with fluconazole-resistant Candida albicans: in vitro study. Mycoses 2011;54(05):e506-e512

16 Iinuma T, Arai Y, Abe Y, et al. Denture wearing during sleep doubles the risk of pneumonia in the very elderly. J Dent Res 2015; 94(3, Suppl):28S-36S

17 Goiato MC, Dos Santos DM, Baptista GT, et al. Effect of thermal cycling and disinfection on colour stability of denture base acrylic resin. Gerodontology 2013;30(04):276-282

18 Vermeulen AH, Keltjens HM, van't Hof MA, Kayser AF. Ten-year evaluation of removable partial dentures: survival rates based on retreatment, not wearing and replacement. J Prosthet Dent 1996; 76(03):267-272

19 Dantas LC, da Silva-Neto JP, Dantas TS, Naves LZ, das Neves FD, da Mota AS. Bacterial adhesion and surface roughness for different clinical techniques for acrylic polymethyl methacrylate. Int J Dent 2016;2016:8685796
20 Vandecandelaere I, Matthijs N, Van Nieuwerburgh F, et al. Assessment of microbial diversity in biofilms recovered from endotracheal tubes using culture dependent and independent approaches. PLoS One 2012;7(06):e38401

21 Kusahara DM, Friedlander LT, Peterlini MA, Pedreira MLG. Oral care and oropharyngeal and tracheal colonization by Gramnegative pathogens in children. Nurs Crit Care 2012;17(03): 115-122

22 Chacko R, Rajan A, Lionel P, Thilagavathi M, Yadav B, Premkumar J. Oral decontamination techniques and ventilator-associated pneumonia. Br J Nurs 2017;26(11):594-599

23 Casey AL, Lambert PA, Elliott TS. Staphylococci. Int J Antimicrob Agents 2007;29(Suppl 3):S23-S32

24 Sampedro GR, Bubeck Wardenburg J. Staphylococcus aureus in the intensive care unit: are these golden grapes ripe for a new approach? J Infect Dis 2017;215(Suppl 1):S64-S70

25 Cliff PR, Sandoe JA, Heritage J, Barton RC. Use of multilocus sequence typing for the investigation of colonisation by Candida albicans in intensive care unit patients. J Hosp Infect 2008;69(01): 24-32

26 Al-Dorzi HM, Sakkijha H, Khan R, et al. Invasive candidiasis in critically ill patients: a prospective cohort study in two tertiary care centers. J Intensive Care Med 2020;35(06):542-553

27 Akpan A, Morgan R. Oral candidiasis. Postgrad Med J 2002;78 (922):455-459

28 Formal SB, Hale TL, Sansonetti PJ. Invasive enteric pathogens. Rev Infect Dis 1983;5(Suppl 4):S702-S707 